\title{
Especialización turística en destinos emergentes latinoamericanos: explorando el algoritmo para un longevo e inclusivo desarrollo socioeconómico
}

\author{
Tourism specialization in emerging Latin American \\ destinations: exploring the algorithm for long-lived and \\ inclusive socio-economic development
}

\author{
Miguel Puig-Cabrera' (1) y Concepción Foronda-Robles²
}

\begin{abstract}
RESUMEN
El notorio crecimiento del turismo en los destinos emergentes genera controversia con respecto a los beneficios socioeconómicos que reporta a la población. Los objetivos de este trabajo son contrastar la capacidad del fenómeno turístico para convertirse en un conductor del desarrollo socioeconómico en los destinos emergentes latinoamericanos y analizar el efecto de la especialización turística sobre las condiciones de vida de la población. Para ello, se ha estimado un modelo econométrico con datos de panel, a partir de una muestra de 20 destinos emergentes entre los años 2005 y 2015. Los resultados muestran que, tanto la especialización turística, como la no turística, tienen un resultado directo y significativo sobre las condiciones de desarrollo socioeconómico de los destinos latinoamericanos, lo que sugiere la conveniencia de diseñar fórmulas de desarrollo socioeconómico basadas en una integración productiva con otros sectores complementarios intensivos en conocimiento y capital. Las aportaciones de este trabajo ofrecen nuevas evidencias empíricas que prueban la pertinencia de las economías latinoamericanas de apostar por el turismo como una actividad dinamizadora de sus economías para generar empleo y riqueza, que además puede servir para dinamizar y apostar por otros sectores complementarios.
\end{abstract}

Palabras clave: Turismo, Desarrollo socioeconómico, Especialización turística, Destino emergente.

\begin{abstract}
The notable growth of tourism in emerging destinations generates controversy regarding the socioeconomic benefits it brings to the population. The objectives of this work are to contrast the capacity of the tourist phenomenon to become a driver of socioeconomic development in emerging Latin American destinations and to analyze the effect of tourism specialization on the living conditions of the population. For this, an econometric model with panel data has been estimated, based on a sample of 20 emerging destinations between 2005 and 2015. The results show that both tourism and non-tourism specialization have a direct and significant on the conditions of socioeconomic development
\end{abstract}

Unidad de Posgrado, Universidad Tecnológica de Santiago (República Dominicana). miguelpuig@docente.utesa.edu Departamento de Geografía Humana, Universidad de Sevilla (España). foronda@us.es 
of Latin American destinations, suggesting the convenience of designing formulas for socioeconomic development based on a productive integration with other complementary sectors intensive in knowledge and capital. The contributions of this work offer new empirical evidence that proves the relevance of Latin American economies to bet on tourism as a dynamic activity of their economies to generate employment and wealth, which can also serve to boost and bet on other complementary sectors.

Keywords: Tourism, Socioeconomic development, Tourism specialization, Emerging destination.

\section{RESUMO}

O notável crescimento do turismo em destinos emergentes gera controvérsia quanto aos benefícios socioeconômicos que traz para a população. Os objetivos deste trabalho são contrastar a capacidade do fenômeno turístico de se tornar um impulsionador do desenvolvimento socioeconômico nos destinos emergentes da América Latina e analisar o efeito da especialização do turismo nas condições de vida da população. Para isso, foi estimado um modelo econométrico com dados em painel, com base em uma amostra de 20 destinos emergentes entre 2005 e 2015 . Os resultados mostram que tanto a especialização em turismo quanto a não-turismo têm direta e indiretamente significativo nas condições de desenvolvimento socioeconômico dos destinos latino-americanos, sugerindo a conveniência de desenhar fórmulas para o desenvolvimento socioeconômico com base em uma integração produtiva com outros setores complementares intensivos em conhecimento e capital. As contribuições deste trabalho oferecem novas evidências empíricas que comprovam a relevância das economias latino-americanas em apostar no turismo como uma atividade dinâmica de suas economias para gerar emprego e riqueza, o que também pode servir para impulsionar e apostar em outros setores complementares.

Palavras-chave: Turismo, Desenvolvimento socioeconômico, Especialização em turismo, Destino emergente.

América recibió 217 millones de llegadas de turistas internacionales en 2018, contando con un crecimiento medio interanual del $3 \%$ para los destinos de América del Sur, y una variación negativa del $2 \%$ en América Central y el Caribe respecto al año anterior (como consecuencia de los huracanes Irma y María en septiembre de 2017). Se estima que para el año 2030 el crecimiento de los destinos emergentes latinoamericanos llevará a que éstos abarquen una cuota de mercado cada vez mayor, sobrepasando a los destinos que actualmente lideran el ranking mundial, y representando una nueva oportunidad para estas economías (OMT, 2019).

En el marco de la Agenda 2030, la OMT (2015) señala el turismo como uno de los principales campos de acción para el cumplimiento de los Objetivos de Desarrollo Sostenible (ODS), vinculándolo de manera directa sobre tres de estas metas: promover el crecimiento económico inclusivo y sostenido (ODS 8); garantizar modalidades de consumo y producción sostenibles (ODS 12) y conservar y utilizar de forma sostenible los recursos marinos (ODS 14).

En el Informe de Turismo para el Desarrollo (OMT, 2018) se puntualiza la necesidad de seguir investigando la conveniencia de apostar por el sector turístico frente a otros sectores productivos para mejorar las condiciones socioeconómicas en destinos emergentes.

El crecimiento del turismo en estos destinos y sus repercusiones sobre las condiciones de vida de la población están originando un creciente dilema en la literatura en torno a las bondades de esta actividad, contando con el respaldo de algunos autores (Bojanic \& Lo, 2016; Koens \& Tho- 
mas, 2015; Telfer \& Sharpley, 2016), frente al rechazo de otros (Gascón, 2015; Scheyvens \& Rusell, 2012). A pesar de esta controversia, el dilema es mayor en lo que respecta a la especialización turística (ET) o economía de "monocultivo" turístico (Chan et al., 2012; Fingini \& Vici, 2010).

En base a la revisión de la literatura y con el objeto de aportar mayor claridad sobre la capacidad del sector turístico para elevar los niveles de desarrollo y bienestar latinoamericano, este trabajo pretende: 1) contrastar la capacidad del fenómeno turístico para convertirse en un conductor del desarrollo socioeconómico en los destinos emergentes; 2) analizar el efecto de la ET sobre las condiciones de vida de la población en estos destinos, en comparación con el desarrollo económico derivado de otros sectores productivos, siendo ésta una de las originalidades del trabajo.

El enfoque utilizado permitirá dar luz a los destinos en función de su grado de "monopolización" turística, con respecto a otros países menos especializados en turismo. Otra de las novedades del trabajo consiste en que se supera la visión estrictamente monetaria, en favor de un desarrollo socioeconómico. Así, se valora este desarrollo desde un enfoque multidimensional (PNUD, 2018) en términos del acceso de la población a un mejor nivel de vida, así como la cobertura de sus necesidades educativas y sanitarias.

El trabajo se organiza de la siguiente manera: tras esta sección introductoria, se realiza una revisión de la literatura que analiza la relación del turismo con el desarrollo socioeconómico, así como los efectos de ET sobre este desarrollo en destinos emergentes. En base a esta revisión, se plantean las hipótesis que son testadas en el trabajo. En las siguientes secciones se presenta la metodología y la base de datos utilizada. A continuación, se muestran los resultados y discusiones. Finalmente, se recalcan las principales conclusiones del trabajo, señalando algunos de los principales factores a tener en cuenta para que el desarrollo turístico contribuya en mayor medida al desarrollo socioeconómico.

\section{Revisión de literatura}

El papel del turismo y su contribución al desarrollo económico han sido abordados extensamente por la literatura académica (Du et al, 2016; Meyer \& Meyer, 2015; Sokhanvar et al., 2018). A pesar de existir cierto consenso en el papel jugado por el sector turístico para la prosperidad socioeconómica, en el caso de los destinos emergentes este efecto positivo continúa generando cierta controversia en el mundo académico.

Algunos autores consideran esta actividad como un importante catalizador hacia la prosperidad socioeconómica (Piergiovanni et al., 2012; Truong et al., 2016), llegando incluso a utilizarse como estrategia para mejorar las condiciones económicas en países desfavorecidos (Croes et al., 2018), y produciendo un efecto multiplicador en la economía.

Por el contrario, otros recalcan que el desarrollo turístico en los destinos emergentes tiende justamente a un continuado empobrecimiento de la población (Gascón, 2015; Scheyvens \& Rusell, 2012). Asimismo, otros trabajos sugieren la no existencia de una clara relación de causalidad entre el crecimiento del sector turístico y el nivel de desarrollo socioeconómico en este tipo de destinos (Llorca-Rodríguez et al., 2017; Mahadevan et al., 2017). 
Esta falta de evidencia empírica en la relación entre el desarrollo turístico y los beneficios económicos derivados del mismo ha generado cierta discusión con respecto a la idoneidad o no de apostar por la ET en un país (Croes et al., 2018; OMT, 2018). Así, mientras algunos autores consideran que el desarrollo turístico representa la principal vía para el crecimiento económico en destinos emergentes (Biagi et al. 2016; Brida et al., 2016), en cambio, Hampton et al. (2018) se muestra más cauto. En esta línea, Croes et al. (2018) enfatizan la necesidad de desarrollar otros sectores distintos para garantizar un mayor crecimiento, criticando las bondades de las economías de "monocultivo turístico".

\section{Causalidad del binomio turismo-desarrollo económico}

Existe un consenso bastante generalizado que atribuye la existencia de una fuerte relación entre el crecimiento del turismo y el crecimiento socioeconómico general de un país (Brida et al., 2016; Njoya \& Seetaram, 2018). Sin embargo, el dilema en la literatura se abre a la hora de atribuir la causalidad de este crecimiento al sector turístico o al desarrollo de otras actividades económicas.

La OMT (2018) señala que el turismo se encuentra directamente asociado al progreso económico en destinos emergentes de dos formas: 1) como causa o factor de crecimiento económico (perspectiva tourism-led); 2) como resultado de un desarrollo previo de otros sectores distintos al turismo (perspectiva economic-led).

Algunos autores señalan que la causalidad del crecimiento económico puede ser atribuida directamente al sector turístico (Chiu \& Yeh, 2017; Zuo \& Huang, 2018), mostrando evidencias empíricas en casos individuales como Irán (Khoshnevis et al., 2017), Líbano (Bassil et al., 2015), Laos (Kyophilavong et al., 2018), Malasia (Solarin, 2018). O bien, en una muestra multipaís para los casos de la región MERCOSUR (Brida et al., 2016), así como destinos que lideran el ranking turístico mundial como México, Francia o Italia, entre otros (Shahzad et al., 2017). Otros, en cambio, niegan la existencia de esa causalidad asociada al turismo (Llorca-Rodríguez et al., 2017; Mahadevan et al., 2017).

Por su parte, también es señalado que es el desarrollo de sectores distintos al turismo el que contribuye al desarrollo socioeconómico de un país, y como desencadenante de este desarrollo, tiene lugar la actividad turística en dicho destino (Lin et al., 2019; Nene \& Taivan, 2017; Sharif et al., 2017).

Sin embargo, otros trabajos sugieren la existencia de una bidireccionalidad, en la que tanto el turismo como el resto de sectores serían conductores de crecimiento económico (Lin et al., 2019; Perles-Ribes, et al., 2017).

\section{Especialización turística versus diversificación productiva}

Independientemente de su direccionalidad, el consenso sobre los efectos positivos del binomio turismo-desarrollo socioeconómico parece ser incuestionable en la literatura. Otro de los grandes debates, más allá de la causalidad de esta relación, gira en torno a la conveniencia de que un país se convierta en una economía de "monocultivo turístico", frente a apostar por sectores distintos al turismo para alcanzar un modelo de desarrollo socioeconómico óptimo. 
Biagi et al. (2017) sugieren que una economía especializada en turismo crece más rápido frente a una economía no turística, especialmente en países en vías de desarrollo. Romao \& Neuts (2017), en cambio, señalan que, en regiones más avanzadas, la ET no es conveniente, siendo el turismo una actividad complementaria al resto de sectores, para contribuir al desarrollo económico.

Marsiglio (2018) indica que la ET no sólo es una efectiva vía para el desarrollo socioeconómico, sino que además facilita que este crecimiento sea inclusivo y "verde".

De Vita \& Kyaw (2017) defienden que la ET es un camino para la prosperidad socioeconómica, aunque se encuentra condicionada por el nivel de desarrollo económico y de la capacidad de absorción del sistema financiero de las economías receptoras. Sus resultados muestran que la relación entre ET y desarrollo socioeconómico es positiva y significativa, aunque los países de ingresos medios y altos parecen ganar más de la ET, que los países de bajos ingresos.

Croes et al. (2018), en cambio, señalan que la ET no guarda una relación directa con el crecimiento económico, e incluso, prueban que la relación de esta variable con la calidad de vida de la población, es negativa, al igual que lo es en cuanto a la inversión.

Marsiglio (2018) sostiene que la ET soporta cambios estructurales y cambios en el uso de recursos, llevando a que un destino asegure beneficios para la población residente, incluso si se encuentra en una fase de estancamiento o declive dentro de su ciclo de vida.

En el caso de los destinos emergentes latinoamericanos, donde el turismo representa una importante parte de su economía, es conveniente estimar hasta qué punto resulta beneficiosa la ET, frente a la diversificación productiva.

En base a este dilema, en la figura $N^{\circ} 1$ se presentan las hipótesis a ser testadas:

Figura No 1

Hipótesis de partida

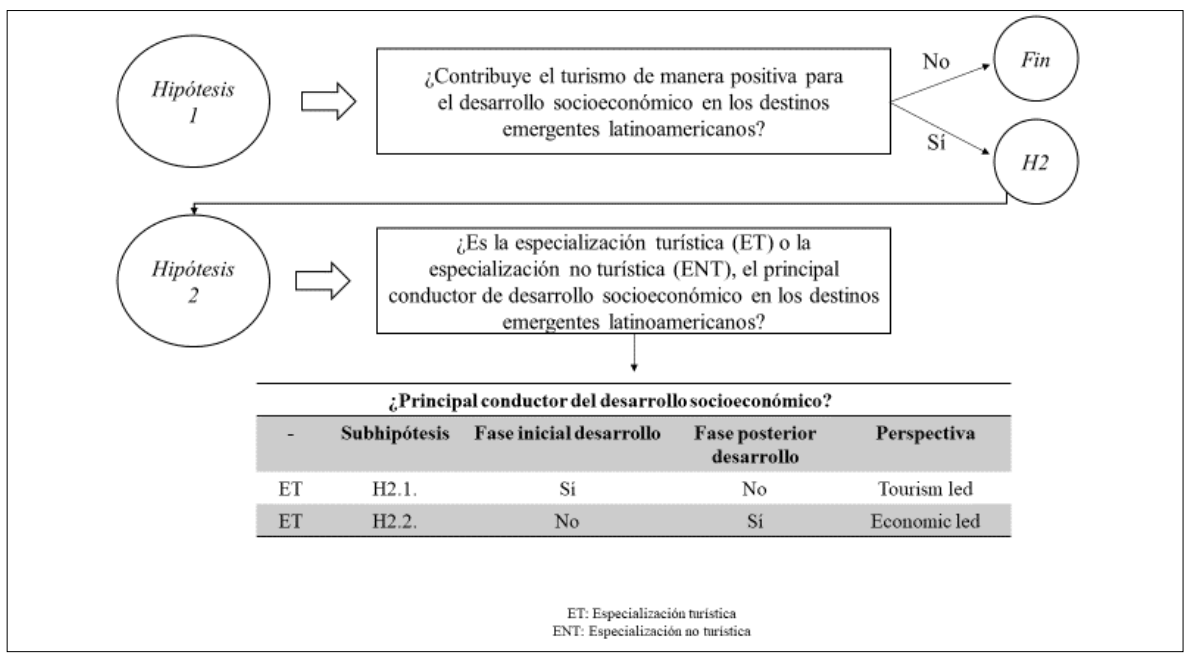

Fuente: Elaboración propia 
H1: El desarrollo del sector turístico en las economías emergentes latinoamericanas en los últimos diez años ha impactado positivamente en los niveles de desarrollo y bienestar de la población.

Sin embargo, en miras de un mayor desarrollo socioeconómico, es conveniente analizar hasta qué punto para estas economías sería recomendable apostar por una especialización turística (ET), frente a la apuesta por una diversificación productiva, es decir, una especialización no turística (ENT):

H2: La ET constituye un factor de desarrollo y mejora del bienestar de la población más potente que el resto de sectores económicos. No obstante, dentro de esta hipótesis caben varios escenarios posibles:

H2.1: A partir de cierto nivel de desarrollo socioeconómico, la contribución de la ENT supera a la ET en el tiempo.

H2.2: A partir de cierto nivel de desarrollo socioeconómico, la contribución de la ET supera a la ENT en el tiempo.

\section{Datos}

\section{Ámbito de estudio}

En este trabajo se analizan datos de panel para 20 destinos emergentes latinoamericanos entre los años 2005-2015 (Figura № 2), considerados a nivel internacional, atendiendo a las publicaciones periódicas del FMI (2016) y la OMT (2019): World Economic Outlook y World Tourism Highlights.

Figura $\mathrm{N}^{\circ} 2$

Ámbito de estudio

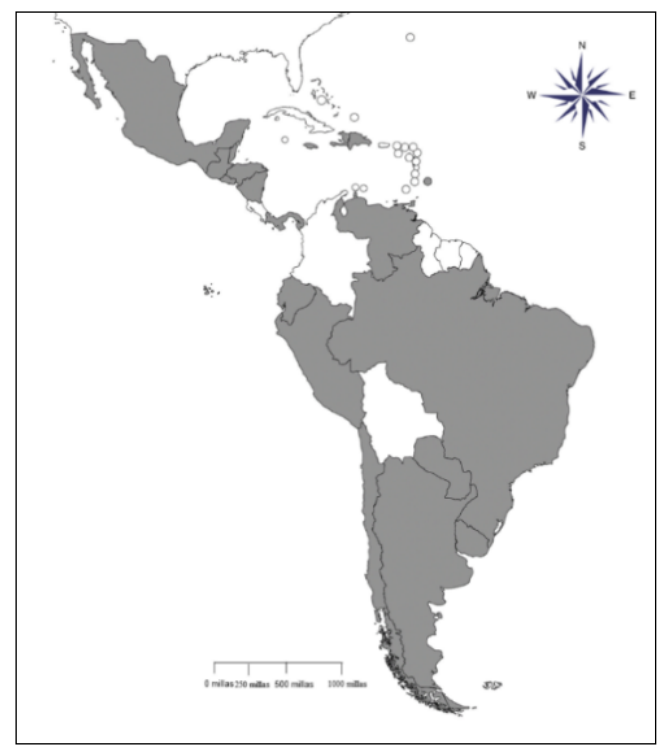

Fuente: Elaboración propia 
Estos destinos se caracterizan por tener al turismo como uno de los principales motores de su economía. Asimismo, las previsiones de la OMT (2019) sugieren que ocuparán las primeras posiciones del ranking mundial, superando así a los destinos tradicionales en el año 2030, coincidiendo con el horizonte de la Agenda 2030 y los ODS (OMT, 2015). La importancia del turismo en estos países, así como el nivel de crecimiento turístico con el que cuentan, se convierten en dos elementos fundamentales para analizar el modelo latinoamericano de desarrollo socioeconómico y turismo, valorando su idoneidad, o en caso contrario, actuar de manera congruente con la situación.

La conformación de la muestra definitiva ha sido realizada en función de la disponibilidad de datos de aquellos países que son clasificados como destinos emergentes y que además pertenecen a la región de Latinoamérica.

Los destinos finales que conforman la muestra son: Argentina; Barbados; Belize; Brasil; Chile; Ecuador; El Salvador; Guatemala; Haití; Honduras; Jamaica; México; Nicaragua; Panamá; Paraguay; Perú; República Dominicana; Trinidad y Tobago; Uruguay y Venezuela.

\section{Descripción de las variables}

Las variables a las que se acude atienden a la medición de los efectos que tiene el turismo sobre las condiciones de vida de la población, comparando tanto la repercusión de la ET como de la ENT (Cuadro No 1):

Cuadro No 1

Datos utilizados en el análisis

\begin{tabular}{|l|l|c|}
\hline Variable & Fuente & $\begin{array}{c}\text { Unidad } \\
\text { Índice }\end{array}$ \\
\hline $\begin{array}{l}\text { Especialización turística (ET) } \\
\text { (logaritmo neperiano) }\end{array}$ & Banco Mundial, 2018; WTTC, 2018 & $\begin{array}{c}\text { Miles \$ a precios } \\
\text { constantes año 2010 }\end{array}$ \\
\hline $\begin{array}{l}\text { Especialización no turística (ENT) } \\
\text { (logaritmo neperiano) }\end{array}$ & Banco Mundial, 2018; WTTC, 2018 & $\begin{array}{c}\text { Miles \$ a precios } \\
\text { constantes año 2010 }\end{array}$ \\
\hline Índice de supervivencia (IS) & Banco Mundial, 2018 & Número de años \\
\hline $\begin{array}{l}\text { Capital humano (CH) } \\
\text { (logaritmo neperiano) }\end{array}$ & Penn World Table, 2018 & Número de años \\
\hline $\begin{array}{l}\text { Libertad política (LP) } \\
\text { (logaritmo neperiano) }\end{array}$ & Freedom House, 2018 & Índice \\
\hline Gasto en sanidad (GS) & Banco Mundial, 2018 & $\%$ \\
\hline
\end{tabular}

Fuente: Elaboración propia

La variable IDH es utilizada para medir el nivel de bienestar alcanzado por la población en el horizonte temporal (2005-2015). Esta variable ha sido obtenida de los Informes de Desarrollo Humano elaborados anualmente por el Programa de las Naciones Unidas para el Desarrollo (PNUD). Este índice mide el desarrollo económico de un país a partir del acceso de la población a un mejor nivel de vida (o bienestar), como la cobertura de sus necesidades educativas (años de escolarización) y sanitarias (esperanza de vida al nacer). Sus valores se comprenden entre 0-1, siendo 0 la situación más próxima al subdesarrollo (bajo nivel de vida, y necesidades educativas y sanitarias 
insatisfechas), y 1 para la situación de máximo desarrollo (elevados niveles de vida y necesidades educativas y sanitarias satisfechas). EI IDH ha sido utilizado por la literatura para vincularse con los efectos socioeconómicos del turismo en un destino (Claveria \& Poluzzi, 2017). Algunas economías como la de Nueva Zelanda ha apostado por este enfoque (Forbes, 2019), pues a pesar del crecimiento socioeconómico, difícilmente será deseable de habitar si existen personas sin hogar, un medio ambiente en degradación, o personas con problemas de salud que no reciben el tratamiento que merecen.

Asimismo, la ET (Chiu \& Yeh, 2017) ha sido calculada a partir del PIB turístico total de la base de datos del Consejo Mundial de Viajes y Turismo (WTTC, 2018) y la población residente en el destino, en función de las estadísticas del Banco Mundial (2018). Esta variable mide la renta turística obtenida por la población en el destino y es expresada en dólares a precios constantes (año base 2010) per cápita, permitiendo cuantificar la contribución individual del turismo sobre las condiciones de vida de la población. Así, puede ser contrastada con la contribución del resto de sectores productivos reportados por la ENT. En definitiva, esta variable puede entenderse como la parte de la producción total de un país en un año determinado que, de manera directa o indirecta, puede ser atribuida al sector turístico.

La ENT (Khan et al., 2017; Stern \& Van Dijk, 2017) supone el conjunto total de bienes y servicios producidos en un país y año determinado, y que no se encuentra vinculado al sector turístico. Esta variable ha sido construida a partir de la diferencia entre el PIB general del país y el PIB turístico total, y el cociente con población total residente en el destino. Se han utilizado datos procedentes del WTTC (2018) y del Banco Mundial (2018) y es expresada en dólares a precios constantes (año base 2010) per cápita.

ET y ENT respectivamente permiten contrastar hasta qué punto en estos destinos es más conveniente una economía de monocultivo turístico, o bien apostar por la diversificación productiva, de cara a la repercusión sobre el bienestar de la población, por lo que la variable dependiente en el análisis siempre es IDH, utilizada como variable proxy del desarrollo socioeconómico (ecuación 1)

Asimismo, se incluyen variables que, si a priori se conocen que inciden de manera positiva sobre sobre el sobre el desarrollo socioeconómico, buscan dotar de mayor validez al modelo: índice de supervivencia (IS), capital humano (CH), libertad política (LP), así como gasto en sanidad (GS).

Conviene realizar un apunte acerca de las variables IS y $\mathrm{CH}$ como predictores de IDH en esta investigación, puesto que, aunque recogen aspectos similares, no suponen una duplicidad en el modelo, y resultan de interés para ser incorporadas dentro del análisis.

La variable IS (Banco Mundial, 2018), a diferencia de la esperanza de vida incluida en el IDH, no mide la edad media a la que llegan los residentes de un determinado país, sino que cuantifica la población que alcanza o cruza el umbral de los 65 años de edad. La inclusión de esta variable se justifica en la escasez de instrumentos normativos de protección para la población anciana que caracteriza a Latinoamérica (PNUD, 2010), a pesar de que la tendencia al envejecimiento en esta región se encuentra en crecimiento (BID, 2018). Por tanto, aunque ambas variables se encuentran significativamente asociadas entre sí, no se produce duplicidad. Esta variable se concibe como una variable que permite medir la calidad de vida de la población en un país (Ridderstaat et al., 
2019), habiendo sido utilizada en otros trabajos de turismo y prosperidad económica para destinos emergentes (Glover \& Prideaux, 2009; Kubickova et al., 2017; Pratt, 2015).

Con respecto a la variable $\mathrm{CH}$, mientras el IDH refleja el pilar de la educación a partir del total de años de escolarización de la población, el CH de la Penn World Table (2018) ha sido extraído considerando los años de educación en relación a la productividad económica del país. Variable empleada en investigaciones sobre turismo y desarrollo (Brida et al., 2016; Stauvermann \& Kumar, 2017).

Por otro lado, la variable LP consiste en un índice elaborado por Freedom House (2018) que establece el nivel de libertad socio-política existente en un país. La situación política se considera clave a la hora de estimar un modelo de desarrollo socioeconómico (Bozkurt et al., 2018), puesto que lo condiciona, especialmente en economías emergentes (Nordin \& Nordin, 2017). Y en el caso del sector turístico, esta cuestión es relevante (Saha et al., 2017), puesto que condiciona en gran medida el hecho de ser visitado o no. Este índice se basa en 25 indicadores relacionados con los derechos políticos y civiles, y se encuentra comprendido entre $0-7$, donde un valor de 0 representa el menor grado de libertad, mientras que un valor de 7 indica el máximo nivel de libertad en el país.

Por último, la variable GS indica el porcentaje del PIB total que un país invierte en sanidad. Esta variable se encuentra expresada en términos porcentuales y ha sido extraída del Banco Mundial (2018). La literatura establece relaciones significativas entre esta variable y el desarrollo económico, tanto en economías desarrolladas, como emergentes (Blanchard \& Giavazzi, 2016). Esta variable también es aludida desde el turismo (Ridderstaat et al., 2019).

En definitiva, las variables utilizadas han sido seleccionadas asegurando su idoneidad para la medición de los efectos de la ET y la ENT sobre el desarrollo socioeconómico de los países de la muestra, incluyendo un vector de variables no turísticas que sirven de referencia en el análisis (ecuación 1).

\section{Análisis descriptivo de las variables}

El Cuadro No 2 muestra los principales estadísticos de las variables que comprenden el estudio, donde las filas "overall" representan el promedio de la muestra de los 20 destinos incluidos en la muestra $(n=20)$ para el periodo 2005-2015 (T=11); las "between" se refieren a la desviación estándar; y las "within" muestran la variación de cada país respecto a su media.

Cuadro $\mathrm{N}^{\circ} 2$

Estadísticos descriptivos

\begin{tabular}{|c|c|c|c|c|c|c|}
\hline \multicolumn{2}{|c|}{ Variable } & Media & $\begin{array}{c}\text { Desviación } \\
\text { estándar }\end{array}$ & Mínimo & Máximo & Observaciones \\
\hline \multirow{3}{*}{$I D H$} & Overall & 0.625 & 0.138 & 0.290 & 0.449 & \\
\cline { 2 - 7 } & Between & & 0.138 & 0.347 & 0.848 & $n=20$ \\
\cline { 2 - 7 } & Within & & 0.196 & 0.555 & 0.682 & $T=11$ \\
\hline \multirow{3}{*}{ LnET } & Overall & 0.0021 & 0.747 & 7.071 & 4.676 & \\
\cline { 2 - 7 } & Between & & 0.214 & 0.657 & 0.462 & $n=20$ \\
\cline { 2 - 7 } & Within & & 0.716 & 6.416 & 4.212 & $T=11$ \\
\hline
\end{tabular}




\begin{tabular}{|c|c|c|c|c|c|c|}
\hline \multicolumn{2}{|c|}{ Variable } & Media & $\begin{array}{c}\text { Desviación } \\
\text { estándar }\end{array}$ & Mínimo & Máximo & Observaciones \\
\hline \multirow{4}{*}{ LnENT } & Overall & 0.010 & 0.628 & 5.985 & 3.280 & \\
\cline { 2 - 7 } & Between & & 0.173 & 0.507 & 0.365 & $n=20$ \\
\cline { 2 - 7 } & Within & & 0.604 & 5.462 & 2.932 & $T=11$ \\
\hline \multirow{4}{*}{ IS } & Overall & 0.0002 & 0.620 & 0.438 & 0.428 & \\
\cline { 2 - 7 } & Between & & 0.014 & 0.035 & 0.044 & $n=20$ \\
\cline { 2 - 7 } & Within & & 0.060 & 0.403 & 0.383 & $T=11$ \\
\hline \multirow{4}{*}{ LnCH } & Overall & 0.0001 & 0.121 & 1.030 & 0.657 & \\
\cline { 2 - 7 } & Between & & 0.035 & 0.094 & 0.071 & $n=20$ \\
\cline { 2 - 7 } & Within & & 0.116 & 0.935 & 0.585 & $T=11$ \\
\hline \multirow{3}{*}{ LnLP } & Overall & 0.0006 & 0.248 & 1.945 & 1.871 & \\
\cline { 2 - 7 } & Between & & 0.072 & 0.194 & 0.187 & $n=20$ \\
\cline { 2 - 7 } & Within & & 0.237 & 1.750 & 1.685 & $T=11$ \\
\hline \multirow{3}{*}{ GS } & Overall & 0.0002 & 0.851 & 7.718 & 4.826 & \\
\cline { 2 - 7 } & Between & & 0.269 & 0.918 & 0.845 & $n=20$ \\
\hline & Within & & 0.807 & 6.799 & 4.140 & $T=11$ \\
\hline
\end{tabular}

Fuente: Elaboración propia

La varianza total "overall" ( $\sigma 2)$ se descompone entre "within" ( $\sigma w 2)$ y "between" ( $\sigma b 2)$. Atendiendo a la variación de las variables, se observa que existe una mayor varianza dentro de los países a lo largo del tiempo ( $\sigma w 2)$, frente a la que tienen los países de la muestra entre sí ( $\sigma b 2):$ IDH cuenta con una $\sigma w 2>\sigma b 2(0.196>0.138)$; para LnET, $\sigma w 2>\sigma b 2(0.716>0.214)$; en el caso de LnENT, $\sigma w 2>\sigma b 2$ ( $0.604>0.173)$; considerando $\mathrm{IS}, \sigma w 2>\sigma b 2(0.060>0.0 .014)$; para $\mathrm{LnCH}, \sigma w 2>\sigma b 2(0.116$ $>0.035)$; en LnLP, $\sigma w 2>\sigma b 2$ (0.237 > 0.072); y para el caso de GS, $\sigma w 2>\sigma b 2(0.807>0.269)$.

El hecho que la varianza entre los países sea mayor que la existente entre los diversos países de la muestra, implica que los destinos objeto de estudio pueden ser analizados de manera conjunta en el análisis.

Siguiendo los principios de un adecuado análisis ANOVA (Kim, 2014), si en el horizonte temporal analizado, la varianza entre países resultara mayor que la varianza dentro de los propios países, se estaría trabajando con submuestras que no son comparables entre sí. 
Figura $\mathrm{N}^{\circ} 3$

Matriz de correlación

IDH-Especialización turística (ET)

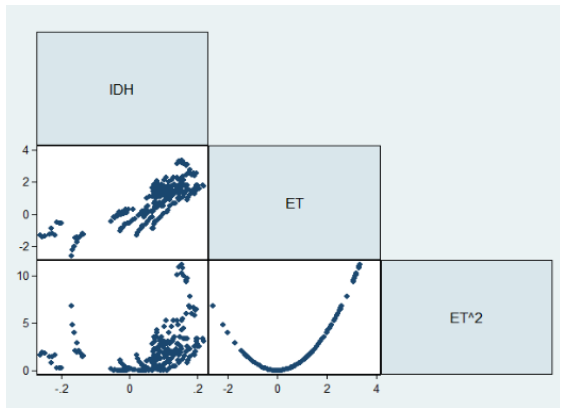

IDH-Especialización no turística (ENT)

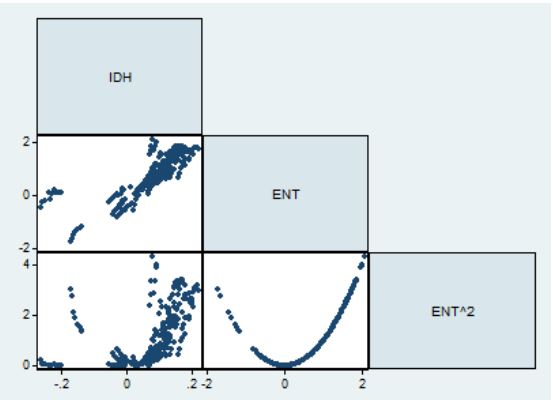

Fuente: Elaboración propia

La figura No 3 muestra la relación entre IDH, ET y ENT a lo largo del periodo 2005-2015. En la matriz se incluyen las transformaciones de ET y ENT a partir de sus desviaciones sobre la media geométrica de la muestra. En ambos casos, se observa que las relaciones son directas, esto supone que, tanto un incremento de ET como de ENT, se encuentra correlacionado con un crecimiento del IDH, de manera lineal y cuadrática. Esto sugiere la existencia de posibles rendimientos a escala entre las relaciones analizadas.

Atendiendo a la tasa de variación media partiendo del año 2005 como base, la variable ET ha experimentado un crecimiento del $26.1 \%$ en el conjunto de la muestra de países latinoamericanos.

En el caso del IDH (toma valores dentro del intervalo 0-1 puntos), éste ha crecido una media de 0.1 puntos.

Asimismo, se observan numerosos casos donde los niveles de ET e IDH crecen conjuntamente, sugiriendo la existencia de una posible correlación positiva, en línea con lo que sugiere la literatura (Biagi et al., 2017), destacando los casos de Argentina, Chile, Costa Rica o Paraguay. Por último, debe hacerse una mención especial a los pequeños estados insulares, siendo reconocidos por ser economías de monocultivo turístico (OMT, 2018), y destacando en este análisis los casos de Haití, República Dominicana o Trinidad y Tobago.

Conocer la existencia de una relación positiva entre IDH y ET supone un buen punto de partida a favor de esta actividad en los destinos emergentes para el presente estudio.

No obstante, se convierte en una prioridad conocer en qué medida el turismo afecta de manera favorable sobre las condiciones de vida de la población, con respecto al bienestar que reporta el resto de sectores, dado que la ENT también muestra una relación directa con IDH.

En este punto del análisis, se enfatiza conocer en qué medida tanto ET como ENT contribuyen al bienestar de la población, en la medida en que ambos guardan una relación directa con IDH. 


\section{Metodología}

Siguiendo el supuesto que el turismo contribuye al crecimiento económico en destinos emergentes (OMT, 2018; Puig-Cabrera \& Foronda-Robles, 2019; Telfer \& Sharpley, 2016), este trabajo se centra en analizar la incidencia del sector turístico sobre las condiciones de vida de la población latinoamericana, y, por ende, sobre el desarrollo socioeconómico en los destinos emergentes. Para ello, se profundiza en este aspecto, tratando de cuantificar la conveniencia de los países objeto de estudio de especializarse en el turismo (ET), frente a diversificar su actividad y apostar por una especialización no turística (ENT). Se parte de un modelo teórico general:

$$
D E_{i t}=f\left(E T_{i t}, E N T_{i t}, C_{i t}\right)
$$

Donde: DE refleja el desarrollo económico de un país; ET representa la especialización turística; ENT abarca todos los sectores distintos al turismo; $\mathrm{C}$ es un vector que recoge todas aquellas variables complementarias que sirven de referencia en el análisis; i es un subíndice que denota los 20 destinos latinoamericanos objeto de estudio y t hace alusión a los años que abarca el estudio (2005-2015).

Partiendo de este modelo general (ecuación 1), se ha estimado una modelización econométrica con objeto de estimar la incidencia de la ET y la ENT sobre las condiciones de vida de la población:

$$
\begin{aligned}
& I D H=\Delta A_{i t}+\beta_{1} E T_{i t}+\beta_{2} E T_{i t}^{2}+\beta_{3} E N T_{i t}+\beta_{4} E N T_{i t}^{2}+\beta_{5} I S_{i t}+\beta_{6} C H_{i t}+\beta_{7} L P_{i t}+\beta_{8} G S_{i t} \\
& +e_{i t}
\end{aligned}
$$

Donde: ET y ENT representan la especialización turística y no turística respectivamente; IS hace referencia al índice de supervivencia; $\mathrm{CH}$ representa el capital humano; LP refleja la libertad política; GS hace referencia al gasto en sanidad; $\Delta A i t=d t$, donde $d t$ representa la suma del efecto individual $\left({ }_{i}\right)$ y temporal $\left({ }_{t}\right)$; y e es el término de error.

Además de la regresión lineal, se ha realizado un análisis ajustado a ecuaciones cuadráticas para profundizar en las relaciones entre la ET y la ENT. El hecho de incluir valores al cuadrado en la ecuación, implica un gran riesgo de inducir multicolinealidad entre las variables del modelo. Para reducir esta multicolinealidad ha sido necesario transformar las variables ET y ENT en términos de desviaciones sobre la media geométrica de la muestra (Azorín et al., 2012). Esto implica que una vez que las variables han sido transformadas, los coeficientes reflejan el valor de la elasticidad del IDH con respecto a ET2 y ENT2.

Se han realizado las correspondientes conversiones logarítmicas (Ln), introduciendo así estacionariedad en el modelo, con el objeto de que la varianza sea constante. Al tratarse de un modelo nivel-log, los coeficientes del modelo (lineales y cuadráticos) corresponden a variables logarítmicas que representan la variación porcentual con respecto a la variable dependiente (Grampella et al., 2016; Villano, 2013). 
Cuadro No 3

Test de raíces unitarias y de cointegración

\begin{tabular}{|c|c|c|c|c|c|c|}
\hline \multirow[b]{3}{*}{ Variable } & \multicolumn{4}{|c|}{ Test Raíces unitarias de Pesaran } & \multicolumn{2}{|c|}{ Test de cointegración de Westerlund } \\
\hline & \multicolumn{2}{|c|}{ Nivel } & \multicolumn{2}{|c|}{ Primera diferencia } & & \\
\hline & $\mathrm{t}$ & p-valor & $\mathrm{t}$ & p-valor & Ratio varianza (RV) & $\mathrm{p}$-valor \\
\hline IDH & -4.106 & 0.073 & -4.840 & 0.000 & \multirow{7}{*}{3.346} & \multirow{7}{*}{0.000} \\
\hline LnET & -1.432 & 0.832 & -5.654 & 0.000 & & \\
\hline LnENT & -1.552 & 0.697 & -5.804 & 0.000 & & \\
\hline LnIS & -0.521 & 1.000 & -4.419 & 0.000 & & \\
\hline $\mathrm{LnCH}$ & -1.047 & 0.992 & -5.352 & 0.000 & & \\
\hline LnLP & 0.304 & 1.000 & -0.394 & 0.000 & & \\
\hline GS & -1.849 & 0.2777 & -5.109 & 0.000 & & \\
\hline
\end{tabular}

Fuente: Elaboración propia

De manera previa a la estimación del modelo planteado, se ha profundizado en un estudio de las propiedades de las series utilizadas (Cuadro $\mathrm{N}^{\circ} 3$ ). Los resultados de los test de dependencia transversal de Pesaran (2004), de raíces unitarias (test CIPS) de Pesaran (2007) y de cointegración de Westerlund (2008) sugieren la pertinencia de estimar el modelo en primeras diferencias $(\Delta)$. Así, la ecuación 2 se reformula de la siguiente manera:

$$
\begin{gathered}
I D H=\Delta A_{i t}+\beta_{1} \Delta L n E T_{i t}+\beta_{2} \Delta L n E T_{i t}^{2}+\beta_{3} \Delta L n E N T_{i t}+\beta_{4} \Delta L n E N T_{i t}^{2}+\beta_{5} \Delta L n I S_{i t}+\beta_{6} \Delta L n C H_{i t} \\
+\beta_{7} \Delta L n L P_{i t}+\beta_{8} \Delta G S_{i t}+e_{i t}
\end{gathered}
$$

La estimación en primeras diferencias permite obtener resultados más robustos que los de un modelo en niveles (Hendry, 1980), especialmente cuando los datos son persistentes (Woolridge, 2006). Esto permite la corrección de la regresión espuria (Granger et al., 2001) del modelo.

La estimación del modelo ha sido realizada a través de Mínimos Cuadrados Ordinarios (MCO) por medio del método de Errores Estándar Corregidos para Panel (PCSE), resolviendo los problemas de correlación contemporánea (Dufour \& Khalaf, 2003), heterocedasticidad y autocorrelación. Los hallazgos de Beck \& Katz (1995) sugieren que el método de PCSE ofrece resultados más precisos que el método de Mínimos Cuadrados Generalizados Factibles (FGLS).

\section{Resultados y discusión}

El Cuadro N. ${ }^{\circ} 4$ muestra los coeficientes lineales y cuadráticos para el modelo de la ecuación 3:

Cuadro No 4

Resultados de las estimaciones

\begin{tabular}{|l|c|c|c|}
\hline & Coeficiente & Error estándar & $\mathbf{P}>|\mathbf{z}|$ \\
\hline LnET & $0.00243^{\star *}$ & 0.0012 & 0.050 \\
\hline LnET2 & $0.0345^{\star \star *}$ & 0.0005 & 0.000 \\
\hline LnENT & $0.0136^{\star * *}$ & 0.0016 & 0.000 \\
\hline
\end{tabular}




\begin{tabular}{|l|c|c|c|}
\hline & Coeficiente & Error estándar & P> $|\mathbf{z}|$ \\
\hline LnENT2 & $0.0027^{* *}$ & 0.0012 & 0.022 \\
\hline LnIS & $0.7015^{\star * *}$ & 0.0139 & 0.000 \\
\hline LnLP & $0.0035^{\star *}$ & 0.0017 & 0.050 \\
\hline LnCH & $0.1724^{\star * *}$ & 0.0055 & 0.000 \\
\hline GS & $0.0014^{* *}$ & 0.0004 & 0.015 \\
\hline R2 & \multicolumn{3}{|c|}{0.977} \\
\hline Wald chi2 & \multicolumn{3}{|c|}{0.0000} \\
\hline Prob>chi2 & \multicolumn{3}{|c|}{0.32} \\
\hline
\end{tabular}

Atendiendo al coeficiente de determinación del modelo (R2=0.9777), se confirma que el $97.7 \%$ de la variación de IDH puede explicarse por las X covariadas. El método PCSE ha permitido corregir la correlación contemporánea, heteroscedasticidad y autocorrelación entre variables, dando lugar a la confirmación y validación del modelo probado en este trabajo.

El test de Wald chi-cuadrado (Prob>chi2 $=0.0000$ ) confirma que la hipótesis nula debe ser rechazada, lo que significa que ninguno de los coeficientes de regresión en el modelo es igual a cero. Por tanto, se concluye que todas las variables incluidas no son simultáneamente igual a cero.

Las variables en el modelo son significativas al 1\% (LnET2, LnENT, IS y LnCH) y al $5 \%$ (LnET, LnENT2, LnLP y GS) respectivamente.

Tratándose de modelos nivel-log, en los que se combinan variables en niveles y logarítmicas, debe tenerse en cuenta que las primeras se expresan en "cambios unitarios", mientras que las segundas se corresponden a variaciones "porcentuales". En este sentido, cuando aumenta una unidad de $\mathrm{x}$, la variable independiente y varía en términos porcentuales, bajo el supuesto de ceteris paribus.

De manera generalizada, se observa que, tanto ET como ENT contribuyen de manera positiva al $\mathrm{IDH}, \mathrm{y}$, por tanto, a las condiciones de vida de la población en los países que conforman la muestra.

En este punto del trabajo se confirma y valida H1. La ET incide de manera positiva sobre las condiciones socioeconómicas de los 20 destinos latinoamericanos analizados. Esto demostraría que el turismo resulta ser un sector que contribuye positivamente al bienestar socioeconómico de la población, tal como sostienen otros trabajos (Njoya \& Seetaram, 2018; Scheyvens \& Russell, 2012). Concretamente, por cada millar adicional de dólares producidos por la ET, el IDH se ve incrementado en un $0.24 \%$. No obstante, la contribución de ENT a IDH es de un incremento del $1.36 \%$ por cada millar de dólares procedente de sectores distintos al turismo, es decir, más de cinco veces el efecto de ET. Sin embargo, a partir de los efectos lineales y cuadráticos de ambas variables, se observa que $\mathrm{ET}^{2}$ (sector turístico) repercute en mayor medida que $\mathrm{ENT}^{2}$ (los sectores distintos al turismo) a este desarrollo socioeconómico. Esto es abordado en profundidad en la siguiente sección.

Con respecto al resto de variables analizadas en el modelo, en línea con lo que sugieren otros autores (Shaker, 2015), por cada punto adicional que se ve aumentado IS, el IDH se incrementa un $70,15 \%$. Esto se traduce en que el número de personas que llegan al umbral de los 65 años favo- 
rece un mayor bienestar socioeconómico en los destinos objeto de estudio. Latinoamérica sufre de una tendencia al envejecimiento, dada la disminución de las tasas mortalidad y el aumento de esperanza de vida. Sin embargo, contar con índices de desarrollo humano inferiores a otras regiones (BID, 2018), trae consigo una situación crítica que requiere de actuación, en la medida en que las políticas públicas dejan en una situación de vulnerabilidad a la población mayor de 65 de años, al no conocer en profundidad su realidad (UNFPA, 2017): condiciones de pobreza más severas que el resto de segmentos de la sociedad; no tener acceso a una pensión de vejez; tener una cobertura de salud limitada (especialmente en población rural y zonas alejadas), o no contar con mecanismos de integración y participación en la formulación de políticas, entre otros factores.

Asimismo, por cada punto adicional de LP, el IDH se ve incrementado un 0,35\%. La situación política siempre ha sido un punto clave para el desarrollo de un país (Fetscherin, \& Stephano, 2016), ya que en función de la estabilidad política pueden desarrollarse sectores como el turismo (al hacer "visitable" un destino), y regularse otras actividades económicas que permitan impulsar un crecimiento socioeconómico sostenido y ordenado. En los destinos latinoamericanos, la regulación del turismo atraviesa dos momentos clave, pasando de una proclamación democrática del turismo (p. e.: vacaciones pagadas), a promover un modelo de desarrollo basado en la dinamización económica, la calidad, la competitividad y la eficiencia (Schenkel, 2019).

Respecto a $\mathrm{CH}$, un año adicional de educación en términos de productividad por persona en los destinos de la muestra, incide con una variación positiva del 17,2\% sobre el IDH. Este alto nivel de significancia de la variable explicativa sobre la explicada se debe a que el factor años de educación está considerada por ambas. Cinnirella \& Streb (2017) respaldan la noción de que la acumulación de capital humano básico y su productividad son cruciales para el desarrollo socioeconómico de un país. Stauverman \& Kumar (2017) concluyen que un aumento en términos de años de educación/productividad del capital humano del sector turístico, genera efectos positivos en el crecimiento de un país.

En referencia a GS, por cada 1\% que éste es incrementado en los destinos, el IDH aumenta un 0,14\%. Bakare \& Olubokun (2011) muestran una relación significativa y directa entre los gastos de atención médica y los económicos. Atilgan et al., (2017) acuden a un estudio de caso para establecer que un aumento del $1 \%$ en el gasto en salud per cápita en un país, conduce a un aumento del $0,434 \%$ en el producto interno bruto per cápita de dicho país.

\section{Los rendimientos de escala para ET y ENT}

En la sección anterior se confirmó que ET incide de manera positiva $(+0,24 \%)$ sobre las condiciones de vida de la población en los destinos objeto de estudio, aunque en menor medida que ENT (+1,36\%). Concretamente, la contribución de ésta última es 1,12\% mayor que la primera.

En el Cuadro $N^{\circ} 4$ se representan las ecuaciones lineal y cuadrática de ET y ENT, sugiriendo la existencia de posibles variaciones en los rendimientos de escala de ambas variables:

En primer lugar, se recalca lo que ha sido descrito anteriormente. La incidencia de la ENT es más acusada que la de ET (0.0136ENT > 0.00243ET), y, por tanto, la ET, aunque incide de manera 
positiva sobre el IDH de los países objeto de estudio, la ENT representa un conductor de crecimiento más efectivo.

No obstante, a partir de las respectivas relaciones cuadráticas de ET y ENT se observa que este patrón de comportamiento se ve alterado, ante la existencia de dos tipos de rendimiento de escala.

Por un lado, ET comienza a ganar peso en su contribución al IDH, en el momento en el que el resto de sectores productivos llega a cierto nivel de desarrollo. En este caso, se trata de una relación cuadrática que origina rendimientos a escala creciente, puesto que un mismo volumen de PIB turístico (ET) comienza a generar "outputs" de mayor escala en los niveles de bienestar de los países de la muestra $(0.0345 \mathrm{ET} 2>0.00243 \mathrm{ET})$, con respecto a la situación inicial. Chiu \& Yeh (2017) probaron que existe una relación no lineal entre turismo y desarrollo económico, mostrando la variabilidad de la contribución del sector turístico a este desarrollo.

Por otro lado, la contribución de ENT al IDH va reduciéndose de manera paulatina (0.0136ENT $>0.0027$ ENT2), produciendo un rendimiento a escala decreciente, puesto que una misma producción procedente de sectores distintos al turismo no reporta los mismos beneficios socioeconómicos que en la situación inicial. Esto conlleva un empoderamiento gradual de la ET (0.0345ET2 $>0.0136$ ENT > 0.0027ENT2). El momento en el que ET comienza a ganar poder con respecto a la ENT se ha denominado "punto de polarización turística", en la medida en que es a partir de ese nivel que el turismo deja de ser una actividad complementaria para el crecimiento, y se convierte en el principal conductor de bienestar socioeconómico para la población de los destinos que conforman la muestra.

Si bien en el apartado anterior se confirmó y validó $\mathrm{H} 1$, en este punto se confirma parcialmente H2. La ET puede ser el principal conductor de desarrollo socioeconómico, pero una vez desarrollados en cierta medida el resto de sectores productivos, confirmando así H2.2., y rechazando, por tanto, H2.1.

Esto coincide con las evidencias reportadas por otros autores (De Vita \& Kyaw, 2017; Hampton et al., 2018), que señalan la relevancia creciente que adquiere ET en una economía, a medida que el nivel de desarrollo del resto de sectores del destino también crece.

En este sentido, ENT ejercería un efecto dinamizador de la economía, dando lugar a un escenario que hiciera más proclive alcanzar un modelo de desarrollo turístico cuya "producción" de bienestar sea cada vez mayor.

Otros autores (Romao \& Neuts, 2017), en cambio, sostienen que las economías de monocultivo turístico tienden a crecer más lentamente que aquellas economías que centran su desarrollo en sectores distintos al turístico.

Bien es cierto que una economía basada en servicios turísticos, se centra en un intensivo uso de recursos que difícilmente generan valor añadido por sí solo. Sin embargo, desarrollar esta actividad permite generar ingresos que pueden reinvertirse en otros sectores que contribuyan a crear conocimiento y tecnología de manera generalizada, y que también revierten así en el propio sector turístico. 
A partir de estos resultados, se convierte en una prioridad para los destinos latinoamericanos, diseñar fórmulas de desarrollo socioeconómico basadas en una integración productiva, que apuesten por un sector intensivo en capital humano como es el turismo, complementado con otros que sean intensivos en conocimiento y/o capital. Por tanto, mientras el sector turístico supone una importante fuente de riqueza y empleo que dinamiza la economía de un país, también supone un mecanismo económico para reinvertir en otras actividades que promueven la inversión en capital y la generación de conocimiento. Esto, a su vez, contribuye a un círculo virtuoso que permitiría al turismo sustentarse en un modelo de desarrollo con mayor capacitación técnica y know-how. Así, la población residente podría ocupar puestos de mayor responsabilidad en los diversos subsectores turísticos, así como ordenar y planificar adecuadamente el destino, en función de las necesidades e intereses locales. Esto implicaría que la propia población local no requiera agentes externos para una adecuada planificación y gestión de la actividad turística, sea a nivel destino, o sea a nivel negocio turístico.

En el año 2030 se estima que estos destinos representen una cuota de mercado mayor que los destinos tradicionales, trayendo consigo importantes flujos de turismo que, de no ser gestionados adecuadamente, podrían acarrear problemas más severos para un óptimo desarrollo socioeconómico que garantice mejores condiciones de vida a la población.

\section{Conclusión}

Este trabajo aporta nueva evidencia empírica acerca de la contribución del turismo al desarroIlo en los destinos emergentes latinoamericanos. Asimismo, analiza los beneficios socioeconómicos que reporta la ET en relación al potencial efecto de ENT.

Se ha probado que los destinos emergentes cuentan con un modelo de desarrollo turístico que favorece el acceso a un mayor nivel de bienestar para la población, confirmando H1.

Por otro lado, los resultados muestran que la contribución de la ET al desarrollo socioeconómico presenta un elevado grado de variabilidad condicionado por el desarrollo de otros sectores. En este sentido, esas actividades económicas son claves para que el turismo, a posteriori, pueda ganar peso en el bienestar socioeconómico. En caso contrario, si el resto de sectores no llegan a desarrollarse, existe el riesgo de que la actividad turística termine siendo sólo un complemento. Así, se confirma parcialmente $\mathrm{H} 2$.

Los hallazgos de este trabajo demuestran la conveniencia para los destinos latinoamericanos de apostar por un modelo que integre al turismo como instrumento de dinamización económica $y$, además, contribuya al desarrollo de otros sectores intensivos en conocimiento y/o capital. Esto garantizaría un modelo cada vez más competitivo basado en un mayor know how.

De cara al horizonte 2030, los resultados muestran la necesidad de evaluar el alcance de los beneficios socioeconómicos que reporta el modelo de desarrollo turístico para garantizar que esa riqueza generada, contribuye a una paulatina reducción de las desigualdades y la exclusión. 
Este trabajo abre nuevas líneas de investigación en relación al binomio turismo y desarrollo socioeconómico, siendo necesario avanzar sobre la repercusión de la especialización turística en la economía (Croes et al., 2018), y analizar si el desarrollo socioeconómico se encuentra más asociado a los recursos naturales y culturales existentes o a su modelo de gestión.

\section{Agradecimientos}

Este articulo forma parte de las investigaciones desarrolladas en el Programa Nacional de Proyectos de Investigación Fundamental titulado "Inteligencia territorial Vs. crecimiento turístico. La planificación y gestión de destinos ante el nuevo ciclo expansivo inmobiliario" (Ref. PGC2018095992-B-I00), financiado por el Ministerio de Ciencia, Innovación y Universidades del Gobierno de España.

\section{Referencias bibliográficas}

ATILGAN, E., KILIC, D., \& ERTUGRUL, H. M. The dynamic relationship between health expenditure and economic growth: is the health-led growth hypothesis valid for Turkey?. The European Journal of Health Economics, 2017, Vol 18, No 5, p. 567-574.

BAKARE, A. A., \& OLUBOKUN, S. Health care expenditure and economic growth in Nigeria: An empirical study. Journal of Emerging Trends in Economics and Management Sciences, 2011, Vol 2, No 2, p. 83-87.

BANCO MUNDIAL. Data. 2018. Washington DC, Estados Unidos, 2018 (Consulta: 03/06/2018). datos.bancomundial.org/

BASSIL, C.; HAMADEH, M.; SAMARA, N. The tourism led growth hypothesis: the Lebanese case. Tourism Review, 2015, Vol. 70, No 1, p. 43-55.

BECK, N., \& KATZ, J. N. What to do (and not to do) with time-series cross-section data. American Political Science Review, 1995, Vol. 89, No. 3, p. 634-647.

BIAGI, B., LADU, M. G., \& ROYUELA, V. Human development and tourism specialization. Evidence from a panel of developed and developing countries. International Journal of Tourism Research, 2017, Vol. 19, No 2, p. 160-178.

BLANCHARD, O., \& GIAVAZZI, F. Rebalancing growth in China: A three-handed approach. The Economic Development in Contemporary China, 2016, p. 49-84.

BOJANIC, D. C., \& LO, M. A comparison of the moderating effect of tourism reliance on the economic development for islands and other countries. Tourism Management, 2016, No 53, p. 207-214.

BOZKURT, E., ALTINER, A., \& TOKTAŞ, Y. Democracy and economic growth: evidence from emerging market economies. Electronic Turkish Studies, 2018, Vol. 13, No 14, p. 15-32. 
BRIDA, J. G., CORTES-JIMENEZ, I., \& PULINA, M. Has the tourism-led growth hypothesis been validated? A literature review. Current Issues in Tourism, 2016, Vol. 19. № 5, p. 394-430.

BUENDIA, J. D., ESTEBAN, M., \& SÁNCHEZ, J. C. Estimación de la renta bruta disponible municipal mediante técnicas de econometría espacial. Un ejercicio de aplicación. Revista de Estudios Regionales, 2012, No 93, p. 119-142.

CHANG, C. L., KHAMKAEW, T., \& MCALEER, M. IV estimation of a panel threshold model of tourism specialization and economic development. Tourism Economics, 2012, Vol. 18, No 1, p. 5-41.

CHIU, Y. B., \& YEH, L. T. The threshold effects of the tourism-led growth hypothesis: Evidence from a cross-sectional model. Journal of Travel Research, 2017, Vol. 56, No 5, p. 625-637.

CHOU, M. C. Does tourism development promote economic growth in transition countries? A panel data analysis. Economic Modelling, 2013, Vol. 33, p. 226-232.

CINNIRELLA, F., \& STREB, J. The role of human capital and innovation in economic development: evidence from post-Malthusian Prussia. Journal of economic growth, 2017, Vol. 22, No 2, p. 193-227.

CLAVERIA, O., \& POLUZZI, A. Positioning and clustering of the world's top tourist destinations by means of dimensionality reduction techniques for categorical data. Journal of Destination Marketing \& Management, 2017, Vol. 6, № 1, p. 22-32.

CROES, R., RIDDERSTAAT, J., \& VAN NIEKERK, M. Connecting quality of life, tourism specialization, and economic growth in small island destinations: The case of Malta. Tourism Management, 2018, Vol. 65, p. 212-223.

DE VITA, G., \& KYAW, K. S. Tourism specialization, absorptive capacity, and economic growth. Journal of Travel Research, 2017, Vol. 56, No 4, p. 423-435.

DU, D., LEW, A. A., \& NG, P. T. Tourism and economic growth. Journal of Travel Research, 2016, Vol. 55 , No 4, p. 454-464.

DUFOUR, J. M., \& KHALAF, L. Exact tests for contemporaneous correlation of disturbances in seemingly unrelated regressions. Journal of Econometrics, 2002, Vol. 106, No 1, p. 143-170.

FETSCHERIN, M., \& STEPHANO, R. M. The medical tourism index: Scale development and validation. Tourism Management, 2016, Vol. 52, p. 539-556.

FIGINI, P., \& VICl, L. Tourism and growth in a cross section of countries. Tourism Economics, 2010, Vol. $16, N^{\circ} 4$, p. 789-805.

FORBES. New Zealand Ditches GDP For Happiness and Wellbeing. Forbes 2019 (Consulta: 11/07/2019). www.forbes.com/sites/jamesellsmoor/2019/07/11/new-zealand-ditches-gdp-for-happiness-and-wellbeing/\#23b368ef1942 
FREEDOM HOUSE. Freedom in the World. Freedom House, 2018. (Consulta: 12/05/2018). freedomhouse.org/report/freedom-world/freedom-world-2018

GASCÓN, J. Pro-poor tourism as a strategy to fight rural poverty: A critique. Journal of Agrarian Change, 2015, Vol. 15, No 4, p. 499-518.

GLOVER, P., \& PRIDEAUX, B. Implications of population ageing for the development of tourism products and destinations. Journal of Vacation Marketing, 2009, Vol. 15, No 1, p. 25-37.

GRAMPELLA, M., MARTINI, G., SCOTTI, D., \& ZAMBON, G. The factors affecting pollution and noise environmental costs of the current aircraft fleet: An econometric analysis. Transportation $R e-$ search Part A: Policy and Practice, 2016, Vol. 92, p. 310-325.

GRANGER IV, C. W., HYUNG, N., \& JEON, Y. Spurious regressions with stationary series. Applied Economics, 2001, Vol. 33, No 7, p. 899-904.

HAMPTON, M. P., JEYACHEYA, J., \& LONG, P. H. Can tourism promote inclusive growth? Supply chains, ownership and employment in Ha Long Bay, Vietnam. The Journal of Development Studies, 2018, Vol. 54, No 2, p. 359-376.

HENDRY, D. F. Econometrics-alchemy or science? Economica, 1980, Vol. 47, No 188, p. 387-406.

KHAN, S. A. R., QIANLI, D., SONGBO, W., ZAMAN, K., \& ZHANG, Y. Travel and tourism competitiveness index: The impact of air transportation, railways transportation, travel and transport services on international inbound and outbound tourism. Journal of Air Transport Management, 2017, Vol. 58, p. 125-134.

$\mathrm{KIM}, \mathrm{H}$. Y. Analysis of variance (ANOVA) comparing means of more than two groups. Restorative dentistry \& endodontics, 2014, 39(1), 74-77.

KOENS, K., \& THOMAS, R. Is small beautiful? Understanding the contribution of small businesses in township tourism to economic development. Development Southern Africa, 2015, Vol. 32, № 3 , p. 320-332.

KUBICKOVA, M., \& LI, H. Tourism competitiveness, government and tourism area life cycle (TALC) model: the evaluation of Costa Rica, Guatemala and Honduras. International Journal of Tourism Research, 2017, Vol. 19, № 2, p. 223-234.

KYOPHILAVONG, P., GALLUP, J. L., CHAROENRAT, T., \& NOZAKI, K. Testing tourism-led growth hypothesis in Laos? Tourism Review, 2018, Vol. 73, No 2, p. 242-251.

LIN, V., YANG, Y., LI, G. Where can tourism-led growth and economy-driven tourism growth occur? Journal of Travel Research, 2019, Vol. 58, No 5, p. 760-773. 
LLORCA-RODRÍGUEZ, C. M., CASAS-JURADO, A. C., \& GARCÍA-FERNÁNDEZ, R. M. Tourism and poverty alleviation: An empirical analysis using panel data on Peru's departments. International Journal of Tourism Research, 2017, Vol. 19, No 6, p. 746-756.

MAHADEVAN, R., AMIR, H., \& NUGROHO, A. Regional impacts of tourism-led growth on poverty and income inequality: A dynamic general equilibrium analysis for Indonesia. Tourism Economics, 2017, Vol. 23, No 3, p. 614-631.

MARSIGLIO, S. On the implications of tourism specialization and structural change in tourism destinations. Tourism Economics, 2018, Vol. 24, No 8, p. 945-962.

MEYER, D. F., \& MEYER, N. The role and impact of tourism on local economic development: a comparative study: tourism and leisure. African Journal for Physical Health Education, Recreation and Dance, 2015, Vol. 21, № 1.1, p. 197-214.

NENE, G., \& TAIVAN, A. Causality between tourism and economic growth: Evidence from Sub Saharan Africa (SSA). The Journal of Developing Areas, 2017, Vol. 51, No 2, p. 155-169.

NJOYA, E. T., \& SEETARAM, N. Tourism contribution to poverty alleviation in Kenya: A dynamic computable general equilibrium analysis. Journal of Travel Research, 2018, Vol. 57, No 4, p. 513524.

NORDIN, N. N., \& NORDIN, N. H. The role of economic freedom in research and development-productivity growth nexus: Study based on different income level on developing countries. Journal of Economic Cooperation \& Development, 2017, Vol. 38, No 1, p. 1-27.

OMT. Tourism and the Sustainable Development Goals. Organización Mundial de Turismo, Madrid, 2015.

OMT. Tourism for Development Vol I. Organización Mundial de Turismo, Madrid, 2018.

OMT. World Tourism Highlights 2018 Edition. Organización Mundial de Turismo, Madrid, 2019.

PENN WORLD TABLE. The Database. University of Groningen, Netherlands, 2018.

PERLES-RIBES, J. F., RAMÓN-RODRÍGUEZ, A. B., RUBIA, A., \& MORENO-IZQUIERDO, L. Is the tourism-led growth hypothesis valid after the global economic and financial crisis? The case of Spain 1957-2014. Tourism Management, 2017, Vol. 61, p. 96-109.

PESARAN, M. H. A simple panel unit root test in the presence of cross-section dependence. Journal of Applied Econometrics, 2007, Vol. 22, No 2, p. 265-312.

PESARAN, M. H. General diagnostic tests for cross section dependence in panels. CESifo Working Paper Series, 2004, n 1229; IZA Discussion Paper No 1240. 
PIERGIOVANNI, R., CARREE, M. A., \& SANTARELLI, E. Creative industries, new business formation, and regional economic growth. Small Business Economics, 2012, Vol. 39, № 3, p. 539560.

PNUD. Human Development Data. Programa de Naciones Unidas para el Desarrollo: Nueva York, EEUU, 2018. (Consulta: 23/05/2018).

PRATT, S. The economic impact of tourism in SIDS. Annals of Tourism Research, 2015, No 52, p. 148-160.

PUIG-CABRERA, M. \& FORONDA-ROBLES, C. Tourism, smallness and insularity: a suitable combination for quality of life in Small Island Developing States (SIDS)? Island Studies Journal, 2019, Vol. $14, N^{\circ} 2$, p. $61-80$.

RIDDERSTAAT, J.; SINGH, D.; DEMICCO, F. The impact of major tourist markets on health tourism spending in the United States. Journal of Destination Marketing \& Management, 2019, Vol. 11, p. 270-280.

ROMAO, J., \& NEUTS, B. Territorial capital, smart tourism specialization and sustainable regional development: Experiences from Europe. Habitat International, 2017, Vol. 68, p. 64-74.

SAHA, S., SU, J. J., \& CAMPBELL, N. Does political and economic freedom matter for inbound tourism? A cross-national panel data estimation. Journal of Travel Research, 2017, Vol. 56, № 2, p. 221-234.

SALAZAR, F. E. Cuantificación del riesgo de incumplimiento en créditos de libre inversión: un ejercicio econométrico para una entidad bancaria del municipio de Popayán, Colombia. Estudios Gerenciales, 2013, Vol. 29, No 129, p. 416-427.

SCHEYVENS, R. \& RUSSELL, M. Tourism and poverty alleviation in Fiji: Comparing the impacts of small- and large-scale tourism enterprises. Journal of Sustainable Tourism, 2012, Vol. 20, № 3, p. 417-436.

SEBELE, L. S. Community-based tourism ventures, benefits and challenges: Khama rhino sanctuary trust, central district, Botswana. Tourism management, 2010, Vol. 31, № 1, p. 136-146.

SEQUEIRA, T. N., \& MAÇÃS NUNES, P. Does tourism influence economic growth? A dynamic panel data approach. Applied Economics, 2008, Vol. 40, No 18, p. 2431-2441.

SHAHZAD, S. J. H., SHAHBAZ, M., FERRER, R., \& KUMAR, R. R. Tourism-led growth hypothesis in the top ten tourist destinations: New evidence using the quantile-on-quantile approach. Tourism Management, 2017, Vol. 60, p. 223-232.

SHAKER, R. R. The spatial distribution of development in Europe and its underlying sustainability correlations. Applied Geography, 2015, Vol. 63, p. 304-314. 
SHARIF, A., SAHA, S., \& LOGANATHAN, N. Does tourism sustain economic growth? Wavelet-based evidence from the United States. Tourism Analysis, 2017, Vol. 22, Nº4, p. 467-482.

SOKHANVAR, A., ÇIFTÇIOĞLU, S., \& JAVID, E. Another look at tourism-economic development nexus. Tourism management perspectives, 2018, No 26, p. 97-106.

SOLARIN, S. A. Does tourism-led growth hypothesis exist in Mauritius? Evidence from disaggregated tourism markets. Current Issues in Tourism, 2018, Vol. 21, No 9, p. 964-969.

STAUVERMANN, P. J., \& KUMAR, R. R. Productivity growth and income in the tourism sector: Role of tourism demand and human capital investment. Tourism Management, 2017, Vol. 61, p. 426-433.

STERN, D. I., \& VAN DIJK, J. Economic growth and global particulate pollution concentrations. Climatic Change, 2017, Vol. 142, No 3-4, p. 391-406.

TELFER, D.J. \& SHARPLEY, R. Tourism and Development in the Developing World. Abingdon. Routledge, 2016.

TRUONG, S. E. A. V. D., SLABBERT, E., \& NGUYEN, V. M. Poverty in tourist paradise? A review of pro-poor tourism in South and South-East Asia. In The Routledge Handbook of Tourism in Asia, Routledge, 2016, p. 121-138.

UNFPA Una mirada sobre el envejecimiento ¿Dónde están varios países latinoamericanos a 15 años del Plan de Acción Internacional de Madrid? 2017. Fondo de Población de las Naciones Unidas, Nueva York, Estados Unidos.

WESTERLUND, J. Panel cointegration tests of the Fisher effect. Journal of Applied Econometrics, 2008, Vol. 23, No 2, p. 193-233.

WOOLDRIDGE, J. M. Introducción a la Econometría: un enfoque moderno. Madrid, Editorial Paraninfo, 2006, p. 401-435.

WTTC. Data Gateway. Consejo Mundial de Viajes y Turismo, Londres, Reino Unido, 2018. (Consulta: 03/06/18). https://tool.wttc.org/.

YAZDI S. K., SALEHI, K. S., SOHEILZAD, M. The relationship between tourism, foreign direct investment and economic growth: evidence from Iran. Current Issues in Tourism, 2017, Vol. 20, № 1, p. $15-26$.

ZUO, B., \& HUANG, S. Revisiting the tourism-led economic growth hypothesis: The case of China. Journal of Travel Research, 2018, Vol. 57, No 2, p. 151-163. 
